\title{
Landau singularities and symbology: one- and two-loop MHV amplitudes in SYM theory
}

\section{Tristan Dennen, Marcus Spradlin and Anastasia Volovich}

Department of Physics, Brown University, Providence RI 02912, U.S.A.

E-mail: tristan_dennen@brown.edu, marcus_spradlin@brown.edu, anastasia_volovich@brown.edu

ABSTRACT: We apply the Landau equations, whose solutions parameterize the locus of possible branch points, to the one- and two-loop Feynman integrals relevant to MHV amplitudes in planar $\mathcal{N}=4$ super-Yang-Mills theory. We then identify which of the Landau singularities appear in the symbols of the amplitudes, and which do not. We observe that all of the symbol entries in the two-loop MHV amplitudes are already present as Landau singularities of one-loop pentagon integrals.

KEYwords: Scattering Amplitudes, Supersymmetric gauge theory

ARXiv EPrint: 1512.07909 


\section{Contents}

1 Introduction 1

2 Lightning review 2

2.1 Landau singularities 2

2.2 One-loop bubbles, triangles, and boxes 3

2.3 Projective geometry and momentum twistors 5

3 One-loop MHV amplitudes $\quad 7$

3.1 The chiral pentagon $\quad 7$

3.2 Landau singularities 8

$\begin{array}{lll}3.3 & \text { Summary } & 9\end{array}$

4 Two-loop MHV amplitudes 10

$\begin{array}{lll}4.1 & \text { The chiral double pentagon } & 10\end{array}$

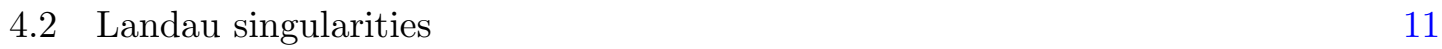

$\begin{array}{lll}4.3 & \text { Summary and discussion } & 13\end{array}$

5 Conclusion and caveats $\quad 14$

\section{Introduction}

A long-standing goal of the S-matrix program is to be able to construct formulas for the scattering amplitudes of a quantum field theory based on general principles and physical constraints. As amplitudes are expected (at finite order in perturbation theory) to be holomorphic functions of the kinematic data, with isolated poles and branch cuts, a thorough understanding of their analytic structure is clearly of paramount importance towards this goal. In 1959 Landau formulated a simple set of equations [1] whose solutions parameterize the locus, in the space of kinematic data, where a given Feynman integral can develop branch points. Subsequent developments are reviewed in the classic book [2], now celebrating its fiftieth year, and more recently in chapter 13 of ref. [3].

In a large variety of theories many of the simplest (and hence best understood) scattering amplitudes can be expressed in terms of a class of generalized polylogarithm functions known also as Chen iterated integrals [4]. Much of the information about the analytic structure of a function $f$ of this type, in particular the locations of its logarithmic branch points, is conveniently encapsulated in an object called the symbol of $f[5,6]$. The entries of a symbol (often called the "letters" of its "symbol alphabet") are various algebraic functions of the kinematic data whose zeros represent branch point singularities (not necessarily on the principal sheet) of the corresponding function. 
As recently pointed out by Maldacena et al. in ref. [7], for amplitudes of generalized polylogarithm type (or, as is commonly the case, of this type together with an algebraic prefactor), there should evidently be a close connection between symbol entries and solutions of the Landau equations. Specifically, the symbol entries appearing in any amplitude (in fact even in any individual Feynman integral) should be such that their zeros specify values of external momenta where solutions of the Landau equations exist. Aspects of this connection have played a role in refs. $[8,9]$, where various one- and two-loop examples were studied.

Although the Landau/symbol connection ought to be quite general, in this paper we focus on examples drawn from $\mathcal{N}=4$ super-Yang-Mills (SYM) theory, specifically the oneand two-loop MHV amplitudes. Although the latter involve double pentagon integrals which are superficially more complicated than the examples studied in refs. [8, 9], our analysis benefits from the fact that the kinematic domain for scattering amplitudes in SYM theory is much simpler than in general field theories.

As we review in section 2, in general the Landau equations admit many families of solutions which are naturally stratified into sets called the leading Landau singularities (LLS), sub-leading Landau singularities (SLLS), sub-sub-leading ( $\left.{ }^{2} L L S\right)$ etc. We introduce these abbreviations to carefully distinguish the Landau singularities of integrals from the (closely related) "leading singularities" of integrands (see for example ref. [10]) which are a key concept in modern work on the mathematical structure of SYM theory. To amplify this point: the leading singularities of an $L$-loop integrand are the points in the $4 L$-dimensional space of loop integration variables where the integrand, a rational function, has nonzero residues (the term is also commonly used to refer to the values of these residues, not their locations); these leading singularities exist for generic values of the external kinematics. In contrast, the Landau singularities of an $n$-point integral or amplitude are the loci in the $3(n-5)$ dimensional space of external kinematic data where the Landau equations admit solutions and where branch points are located. Note that there is no canonical way to distinguish between the leading, sub-leading, etc. Landau singularities of an amplitude - it may depend on the particular representation of the amplitude in terms of Feynman integrals. We therefore refer to the LLS, SLLS, etc. collectively as "the Landau singularities."

Section 2 contains a lightning review of the Landau equations, the projective geometry used for describing the kinematics of SYM theory, and the Landau singularities for various simple one-loop integrals. In section 3 we tabulate the Landau singularities of the chiral pentagon integral, which forms the basic building block for all one-loop MHV amplitudes. We find an exact correspondence between the SLLS and S $^{2}$ LLS and the symbol entries of this integral. The double pentagon integral, in terms of which all two-loop MHV amplitudes are expressed, is analyzed in section 4. Finally we conclude with a discussion of our results and questions for further research.

\section{Lightning review}

\subsection{Landau singularities}

The Landau equations for a given Feynman integral are a set of kinematic constraints that are necessary for the appearance of a pole or branch point in the integrated function (as a 
function of external kinematics and masses). There are multiple ways to formulate them (see ref. [1] for a thorough review), although in this paper we stick to the formulation from the Feynman parameterized integral, where the integrand is a function of loop momenta and Feynman parameters, $\left(\ell_{r}^{\mu}, \alpha_{i}\right)$ :

$$
I=c \int \prod_{r=1}^{L} d^{D} \ell_{r} \int_{\alpha_{i} \geq 0} d^{\nu} \alpha \delta\left(1-\sum_{i=1}^{\nu} \alpha_{i}\right) \frac{\mathcal{N}\left(\ell_{r}^{\mu}, p_{i}^{\mu}, \ldots\right)}{\mathcal{D}^{\nu}}
$$

with

$$
\mathcal{D}=\sum_{i=1}^{\nu} \alpha_{i}\left(q_{i}^{2}-m_{i}^{2}\right)
$$

and $c$ a prefactor that will not enter into our discussion. Here, $q_{i}^{\mu}$ is the momentum flowing along propagator $i, p_{i}^{\mu}$ are external momenta, and $\mathcal{N}$ is some numerator function of the kinematic data. There are two distinct situations in which $I$ can develop a singularity or branch point:

1. The surface $\mathcal{D}=0$ pinches the integration contour in all $\left(\ell_{r}, \alpha_{i}\right)$ simultaneously. The kinematic locations at which this happens are called "Leading Landau Singularities" (LLS).

2. The surface $\mathcal{D}=0$ hits the boundary of the integration contour, at $\alpha_{i}=0$ for some subset of the Feynman parameters, and pinches the contour in all other variables. These are called "Non-leading Landau Singularities," which we stratify into "Subleading" (SLLS), "Sub-sub-leading," ( $\mathrm{S}^{2} \mathrm{LLS}$ ) and so forth, according to how many of the $\alpha_{i}$ are vanishing.

Although we do not review the derivation here, these two situations are captured by the following set of simultaneous equations:

$$
\begin{aligned}
\sum_{i \in \text { loop }} \alpha_{i} q_{i}^{\mu} & =0 \quad \forall \text { loops } \\
\alpha_{i}\left(q_{i}^{2}-m_{i}^{2}\right) & =0 \quad \forall i
\end{aligned}
$$

On the principal sheet, the integration in $\ell_{r}^{\mu}$ and $\alpha_{i}$ in (2.1) is taken over the real axis, with $\alpha_{i} \geq 0$. Branch points on the principal sheet require the solution to (2.3) and (2.4) to pinch this contour. When discussing symbol entries, however, we are also interested in branch points on higher sheets, which are exposed by analytically continuing (2.1) to generic contours. Therefore, throughout this paper we will look more generally for solutions to $(2.3)$ and (2.4) with $\ell_{r}^{\mu}, \alpha_{i} \in \mathbb{C}$.

\subsection{One-loop bubbles, triangles, and boxes}

The Landau equations (2.3) and (2.4) are easily solved for one-loop bubble, triangle, and box integrals in four dimensions. Equation (2.4) puts all of the propagators on-shell, with 


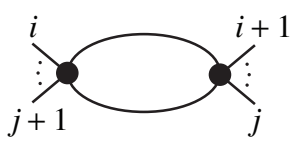

(a)

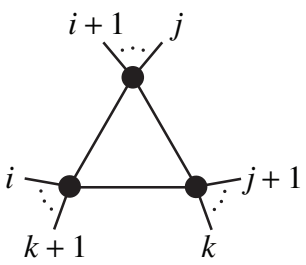

(b)

Figure 1. The scalar bubble and triangle integrals. All propagators are taken to be massless.

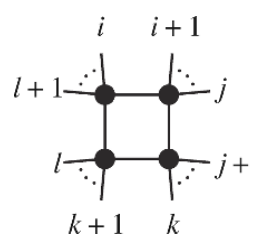

(a)

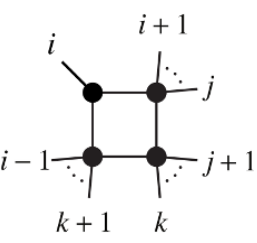

(b)

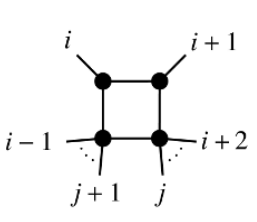

(c)

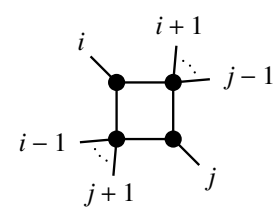

(d)

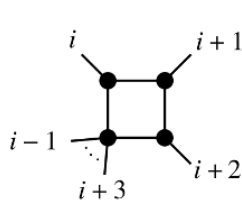

(e)

Figure 2. The scalar box integral and its degenerations. All propagators are taken to be massless.

no constraints on external kinematics, while the solvability of the loop rule (2.3) gives a determinantal constraint after contracting with each of the propagator momenta $q_{i}^{\mu}$.

For the bubble and triangle integrals shown in figure 1, the locations of the LLS are given by

$$
\begin{array}{cl}
\text { Bubble: } & 0=x_{i j}^{2}, \\
\text { Triangle: } & 0=x_{i j}^{2} x_{j k}^{2} x_{i k}^{2},
\end{array}
$$

where the region momenta $x_{i}^{\mu}$ are defined through $p_{i}^{\mu}=x_{i}^{\mu}-x_{i-1}^{\mu}$ and $x_{i j}^{2} \equiv\left(x_{i}-x_{j}\right)^{2}$. In degenerations of the triangle integral when one or more of the corners is on-shell, (2.6) is trivially satisfied for any kinematics. In four dimensions, this is often indicative of the presence of an infrared divergence. More generally, any infrared divergent integral will have either LLS or some $\mathrm{S}^{k} \mathrm{LLS}$ at unconstrained kinematics.

The scalar box integrals are shown in figure 2. As is well known [1], they have the following LLS:

$$
\begin{array}{ll}
\operatorname{Box}(\mathrm{a}): & 0=\left(x_{i j}^{2} x_{k l}^{2}-x_{i k}^{2} x_{j l}^{2}+x_{i l}^{2} x_{j k}^{2}\right)^{2}-4 x_{i j}^{2} x_{j k}^{2} x_{k l}^{2} x_{i l}^{2} \\
\operatorname{Box}(\mathrm{b}): & 0=\langle i(i-1, i+1)(j, j+1)(k, k+1)\rangle \\
\operatorname{Box}(\mathrm{c}): & 0=\langle i-1 i i+1 i+2\rangle\langle i i+1 j j+1\rangle \\
\operatorname{Box}(\mathrm{d}): & 0=\langle i \bar{j}\rangle\langle\bar{i} j\rangle \\
\operatorname{Box}(\mathrm{e}): & 0=\langle i-1 i i+1 i+2\rangle\langle i i+1 i+2 i+3\rangle
\end{array}
$$

The LLS for (b)-(e) are merely degenerations of (a), and we have written them using momentum twistors (reviewed in the next subsection) for easy comparison with the results of the remainder of this paper. The infrared divergences in boxes (b)-(e) are indicated 
by trivial vanishing of some triangle-like SLLS. Inspection of explicit formulas for these integrals [11] reveals a number of branch points not captured by the Landau singularities; we will postpone discussion of these other branch points until section 5 .

\subsection{Projective geometry and momentum twistors}

We begin by reviewing some essential facts about momentum twistor variables [12], which very conveniently parameterize the kinematic configuration space for massless scattering in planar gauge theories, where (for each color-ordered partial amplitude) the particles are endowed with a specific cyclic ordering. We will see that momentum twistors enormously simplify the problem of analyzing the Landau equations in such theories, for the same reason that they simplify the analysis of leading singularities of the integrand (see ref. [13] for several examples of such calculations).

Momentum twistors are based on the correspondence between null rays in (complexified) Minkowski space and points in twistor space $\left(\mathbb{P}^{3}\right)$; or equivalently, between (complex) lines in $\mathbb{P}^{3}$ and points in Minkowski space. We use $Z_{A}, Z_{B}$, etc. to denote points in $\mathbb{P}^{3}$, which may be represented using four-component homogeneous coordinates $Z_{A}=\left(Z_{A}^{1}, Z_{A}^{2}, Z_{A}^{3}, Z_{A}^{4}\right)$ subject to the identification $Z_{A} \sim t Z_{A}$ for any nonzero complex number $t$. We use $(A, B)$ to denote the line in $\mathbb{P}^{3}$ containing two given points $Z_{A}, Z_{B}$. Similarly, $(A, B, C)$ denotes the plane containing the three points $Z_{A}, Z_{B}, Z_{C}$, and $(A, B, C) \cap(D, E, F)$ denotes the line where this plane intersects the plane $(D, E, F)$.

Treating the homogeneous coordinates of each momentum twistor as a vector in $\mathbb{C}^{4}$, there is a natural $\mathrm{SL}(4, \mathbb{C})$ invariant denoted by

$$
\langle A B C D\rangle \equiv \epsilon_{I J K L} Z_{A}^{I} Z_{B}^{J} Z_{C}^{K} Z_{D}^{L} .
$$

We will often be interested to have a geometric understanding of the locus where such fourbrackets might vanish, which can be pictured in several ways. For example, $\langle A B C D\rangle=0$ only if the two lines $(A, B)$ and $(C, D)$ intersect, or equivalently if the lines $(A, C),(B, D)$ intersect, or if the point $A$ lies in the plane $(B, C, D)$, or if the point $C$ lies on the plane $(A, B, D)$, etc. Computations of four-brackets involving intersections may be carried out explicitly with the rule

$$
\langle(A, B, C) \cap(D, E, F) G H\rangle=\langle A B C G\rangle\langle D E F H\rangle-\langle A B C H\rangle\langle D E F G\rangle .
$$

This is manifestly antisymmetric under exchanging any two of the points specifying the plane $(A, B, C)$, or of any two in $(D, E, F)$, or under exchanging the two planes. In case the two planes are specified with one common point, say $F=C$, it is convenient to use the shorthand notation

$$
\langle C(A, B)(D, E)(G, H)\rangle \equiv\langle(A, B, C) \cap(D, E, C) G H\rangle .
$$

This quantity is also manifestly antisymmetric under exchanging $A \leftrightarrow B, D \leftrightarrow E$, or $G \leftrightarrow H$. Moreover, and less obviously, is also fully antisymmetric under exchange of any of the three lines $(A, B),(D, E),(G, H)$. This may be seen with the help of the Schouten identity

$$
\langle A B C D\rangle Z_{E}+\langle B C D E\rangle Z_{A}+\langle C D E A\rangle Z_{B}+\langle D E A B\rangle Z_{C}+\langle E A B C\rangle Z_{D}=0 .
$$




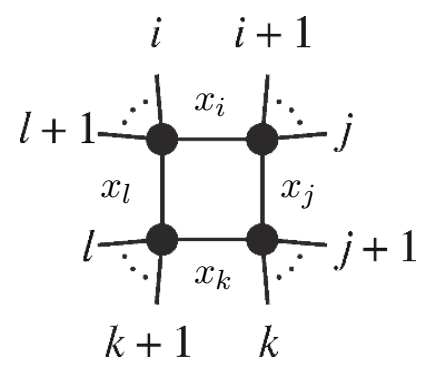

Figure 3. The four-mass box integral.

Now we turn to Hodges' construction [12]. To gain a working understanding of this correspondence it is instructive to take a look at an explicit example, such as the one-loop four-mass box integral shown in figure 3. In a Feynman diagram it is standard to label each edge according to the momentum carried by the associated propagator: $q_{1}, q_{2}$, etc. In a planar diagram it is equally appropriate to label the edges by dual momenta (or region momenta): $x_{1}, x_{2}$, etc. If two faces labeled $x_{a}$ and $x_{b}$ share an edge $q_{c}$, then the momentum running along that edge is $q_{c}=x_{a}-x_{b}$ (an overall orientation must be specified so that the sign of each $q$ in the diagram is consistent with momentum conservation at each vertex).

The external legs in figure 3 are labeled $1, \ldots, n$ where $n$ is the total number of particles. The kinematic data needed to specify the scattering configuration consists of either the collection $p_{1}, \ldots, p_{n}$ of (incoming) momenta of these particles, or a collection $Z_{1}, \ldots, Z_{n}$ of $n$ momentum twistors in $\mathbb{P}^{3}$. The former are related to the dual momenta by $p_{a}=x_{a}-x_{a-1}$. It follows that the four external face variables labeled $x_{i}, x_{j}, x_{k}, x_{l}$ in figure 3 are related to the external momenta by $\left(x_{i}-x_{j}\right)^{2}=\left(p_{i+1}+p_{i+2}+\cdots+p_{j}\right)^{2}$, etc. Hodges' construction for $n$-point kinematics associates the point $x_{a}$ in Minkowski space to the line $(a, a+1)$ in $\mathbb{P}^{3}$ containing the two points $Z_{a}, Z_{a+1}$. One final standard notation worth mentioning is that $\bar{a}$ denotes the plane $(a-1, a, a+1)$.

If $x, y$ are points in Minkowski space associated to two lines $(A, B),(C, D)$ in $\mathbb{P}^{3}$, then their dual spacetime separation may be computed from the formula

$$
(x-y)^{2}=\frac{\langle A B C D\rangle}{\langle I A B\rangle\langle I C D\rangle},
$$

where $I$ is the "infinity twistor" - the line in twistor space associated to the dual spacetime point at spatial infinity. The denominator factors are necessary in order to obtain the flat Minkowski metric in $x$ space, but we will henceforth ignore them as they always drop out of any dual conformal invariant result.

To carry out the integration for the Feynman diagram shown in figure 3 we should associate some dual momentum $x$ to the interior face of the diagram, and then integrate the product of the four propagators $1 /\left(x-x_{i}\right)^{2}$, etc., over $d^{4} x$. In momentum twistor space, the point $x$ is represented by a line $(A, B)$, and we must integrate the product of the four propagators $1 /\langle A B i i+1\rangle$, etc., over the space of lines in $\mathbb{P}^{3}$. Details about how to construct the measure of integration may be found in ref. [13]. Note that the singularity in the propagator $1 /\left(x-x_{i}\right)^{2}$ which arises when $x$ becomes null separated from $x_{i}$ is manifested in momentum twistor space as the singularity in $1 /\langle A B i i+1\rangle$ when the line $(A, B)$ intersects the line $(i, i+1)$. 


\section{One-loop MHV amplitudes}

We now turn our attention to the chiral pentagon integral, which is the basic building block for one-loop MHV amplitudes. This analysis is extremely simple, but it is instructive to go through it carefully as a warm-up for the following section.

\subsection{The chiral pentagon}

The one-loop MHV amplitude for $n$ particles in SYM theory may be expressed as [13]

$$
\frac{\mathcal{A}_{\mathrm{MHV}}^{1-\text { loop }}}{\mathcal{A}_{\mathrm{MHV}}^{\text {tree }}}=\int_{A B} \sum_{1<i<j<n}
$$

in terms of the chiral pentagon integrand

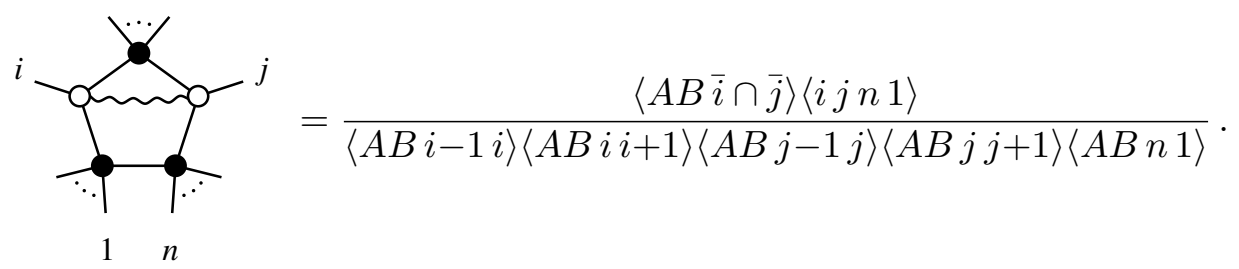

It is useful to recall several comments from ref. [13]. First of all, the full integrand in (3.1) is cyclic invariant despite the appearance of the apparently preferred line $(n, 1)$ on the bottom edge of the pentagon; the formula would be equally valid if $(n, 1)$ were replaced by $(k, k+1)$ for any $k$ (and the summation taken over $k+1<i<j<k)$.

Second, the numerator factors in (3.2) are specially chosen so that all of its leading singularities are normalized to 1 . In fact it would not be inappropriate to say that half of them are 1 and half of them are 0: the scalar pentagon integral has twice as many non-zero leading singularities as the chiral pentagon, but the numerator factors in (3.2) vanish on half of them.

Third, for generic $i$ and $j$ the chiral pentagon integral (3.2) is infrared finite; the numerator factor $\langle A B \bar{i} \cap \bar{j}\rangle$ softens the behavior of the integral precisely in the regions of integration where soft or collinear divergences might appear. This cancellation fails only for certain boundary terms in the sum (specifically, when $i=2$ or $j-i=1$ or $j=n-1$ ) in which case the pentagon degenerates to an IR divergent box integral. Henceforth we ignore these degenerate cases since the box integrals were already reviewed in the previous section.

An explicit formula for the chiral integral was obtained in ref. [13]:

$$
\int_{A B}
$$


in terms of the dual spacetime cross-ratio

$$
u_{i, j, k, l}=\frac{\langle i i+1 j j+1\rangle\langle k k+1 l l+1\rangle}{\langle l l+1 j j+1\rangle\langle k k+1 i i+1\rangle}=\frac{x_{i j}^{2} x_{k l}^{2}}{x_{l j}^{2} x_{k i}^{2}} .
$$

From this explicit result we can easily read off the letters appearing in the symbol of the chiral pentagon. It is already apparent from (3.3) that only the dual spacetime distances $x_{a b}^{2}$ can appear in the first entry of the symbol, reflecting the physically allowed branch points for a scattering amplitude on the physical sheet [14]. In terms of momentum twistors, the 8 letters that appear in the first entry are

$$
\begin{array}{llll}
\langle i-1 i j-1 j\rangle, & \langle i-1 i j j+1\rangle, & \langle i-1 i n 1\rangle, & \langle i i+1 j-1 j\rangle, \\
\langle i i+1 j j+1\rangle, & \langle i i+1 n 1\rangle, & \langle j-1 j n 1\rangle, & \langle j j+1 n 1\rangle .
\end{array}
$$

Six additional letters make an appearance only in the second entry of the symbol:

$$
\begin{array}{lll}
\langle\bar{i} j\rangle, & \langle i(i-1, i+1)(j, j+1)(n, 1)\rangle, & \langle i(i-1, i+1)(j-1, j)(n, 1)\rangle, \\
\langle\bar{j} i\rangle, & \langle j(j-1, j+1)(i, i+1)(n, 1)\rangle, & \langle j(j-1, j+1)(i-1, i)(n, 1)\rangle .
\end{array}
$$

Four of the letters listed in (3.5) also can appear in the second entry of the symbol: $\langle i-1 i n 1\rangle,\langle i i+1 n 1\rangle,\langle j-1 j n 1\rangle$ and $\langle j j+1 n 1\rangle$.

\subsection{Landau singularities}

To find the LLS of the pentagon, one must solve the Landau equations

$$
\begin{gathered}
\alpha_{1}\langle A B i-1 i\rangle=\alpha_{2}\langle A B i i+1\rangle=\alpha_{3}\langle A B j-1 j\rangle=\alpha_{4}\langle A B j j+1\rangle=\alpha_{5}\langle A B n 1\rangle=0, \\
\alpha_{1}\left(x_{A B}-x_{i-1}\right)+\alpha_{2}\left(x_{A B}-x_{i}\right)+\alpha_{3}\left(x_{A B}-x_{j-1}\right)+\alpha_{4}\left(x_{A B}-x_{j}\right)+\alpha_{5}\left(x_{A B}-x_{n}\right)=0
\end{gathered}
$$

for all five $\alpha_{i}$ being nonzero. The equation on the second line of (3.7) is content-free in this case - it tells us to find a vanishing linear combination of five four-component vectors, which is always possible as long as none of the vectors are zero.

The first four equations tell us to find lines $(A, B)$ which intersect the four given lines $(i-1, i),(i, i+1),(j-1, j)$ and $(j, j+1)$. For generic $i, j$ (as we have assumed) there are precisely two solutions to this Schubert problem [13]:

$$
(A, B)=(i, j) \quad \text { or } \quad(A, B)=\bar{i} \cap \bar{j} .
$$

Geometrically this is clear: we can either take $(A, B)$ to be the line $(i, j)$ which contains the two points $Z_{i}, Z_{j}$, or we can take $(A, B)$ to be the intersection of the planes $(i-1, i, i+1)$ and $(j-1, j, j+1)$.

It only remains to solve the equation $\langle A B n 1\rangle=0$, but upon plugging in the solution (3.8) this becomes a constraint on the external kinematics:

$$
\langle i j n 1\rangle\langle n 1 \bar{i} \cap \bar{j}\rangle=0 \text {. }
$$

To conclude: solutions of the Landau equations (3.7) with all $\alpha_{i} \neq 0$ exist only on the locus in kinematic space where (3.9) is satisfied. 
The SLLS of the pentagon are found by solving the Landau equations (3.7) with four of the five $\alpha$ 's being nonzero. Each case amounts to a degeneration of the pentagon to a box, so we can simply transcribe the results of the previous solution. For vanishing $\alpha_{1}, \alpha_{2}$, $\alpha_{3}, \alpha_{4}$ or $\alpha_{5}$ we find respectively that the SLLS lie on the loci:

(SLLS)

$$
\begin{aligned}
& \langle j(j-1, j+1)(i, i+1)(n, 1)\rangle=0, \\
& \langle j(j-1, j+1)(i-1, i)(n, 1)\rangle=0, \\
& \langle i(i-1, i+1)(j, j+1)(n, 1)\rangle=0, \\
& \langle i(i-1, i+1)(j-1, j)(n, 1)\rangle=0, \\
& \langle i j\rangle\langle i \bar{j}\rangle=0 .
\end{aligned}
$$

The $\mathrm{S}^{2}$ LLS are given by solutions of (3.7) with only three nonzero $\alpha$ 's, which correspond to degenerations of the pentagon to various triangles. The four non-trivial cases, arising from three-mass triangles, are

$\left(\mathbf{S}^{2} \mathbf{L L S}\right)$

$$
\begin{aligned}
& \langle i-1 i j-1 j\rangle\langle j-1 j n 1\rangle\langle n 1 i-1 i\rangle=0, \\
& \langle i i+1 j-1 j\rangle\langle j-1 j n 1\rangle\langle n 1 i i+1\rangle=0, \\
& \langle i-1 i j j+1\rangle\langle j j+1 n 1\rangle\langle n 1 i-1 i\rangle=0, \\
& \langle i i+1 j j+1\rangle\langle j j+1 n 1\rangle\langle n 1 i i+1\rangle=0 .
\end{aligned}
$$

Degenerations which lead to two-mass triangles give solutions of the Landau equations for all kinematics, as reviewed in the previous section. These singularities, in the case of the scalar pentagon, are indicative of the soft and collinear IR singularities of the integral. We know however that (for generic $i, j$, as always) the numerator factor in (3.2) eliminates these singularities.

We could go one step further, down to bubbles, but this provides no new information: all bubbles are either fully singular or have Landau singularities on the vanishing loci of brackets which already appear in (3.11).

\subsection{Summary}

We have tabulated all Landau singularities of the pentagon integral. Some sufficiently degenerate singularities exist for all kinematics. Often such singularities are indicative of IR divergences, but we know that for this particular integral (and for generic $i, j$ ) these are canceled by the numerator factor in (3.2). Let us emphasize that except for appealing to this fact, the analysis of the previous section applies to the scalar pentagon integral just as well as the chiral integral, since the Landau equations by definition only know about the propagator structure of a diagram.

The singularities that exist only on various nontrivial submanifolds of kinematic space are indicated in equations (3.9), (3.10) and (3.11). Upon comparison with equations (3.5) and (3.6) we notice a striking pattern: sub-sub-leading Landau singularities (3.11) exist only on the loci where the leftmost symbol entries (3.5) vanish, while sub-leading singularities (3.10) appear on a different set of loci corresponding to the locations where the 
second-entry symbol entries (3.6) vanish. (However let us not forget that (3.6) only lists the new letters which start to appear in the second entry.)

What about the LLS, which lives on the locus $\langle i j n 1\rangle\langle n 1 \bar{i} \cap \bar{j}\rangle=0$ ? This quantity indeed makes an appearance in the overall prefactor in the scalar pentagon integral, which evaluates (see for example ref. [15]) schematically to $1 / \Delta$ times a transcendental function of uniform weight 2 , where $\Delta \propto\langle i \bar{j}\rangle\langle\bar{i} j\rangle\langle i j n 1\rangle\langle n 1 \bar{i} \cap \bar{j}\rangle$. The chiral pentagon, however, is a pure integral: as is evident from (3.3), it evaluates to a transcendental function with no algebraic prefactor. This cancellation is achieved by the carefully chosen numerator of the chiral pentagon, and it is intimately connected with the fact that its integrand has "unit leading singularities," as emphasized in ref. [13].

Evidently this is a happy example where there is a very clear separation between the LLS, which tell us only about the overall algebraic singularities of the amplitude (and which actually end up washed out by the fact that the leading singularities of the integrand are normalized to 1), and the sub-(sub-)leading Landau singularities which probe past the prefactor and into the symbol. Let us recall that MHV amplitudes are expected to evaluate to pure transcendental functions, with no algebraic prefactors (other than the tree-level MHV amplitude indicated on the left-hand side of (3.1)) to all orders in perturbation theory [16].

\section{Two-loop MHV amplitudes}

We now turn our attention to the chiral double pentagon integral, which is the basic building block for two-loop MHV amplitudes.

\subsection{The chiral double pentagon}

The two-loop MHV amplitude for $n$ particles in SYM theory may be expressed as [13]

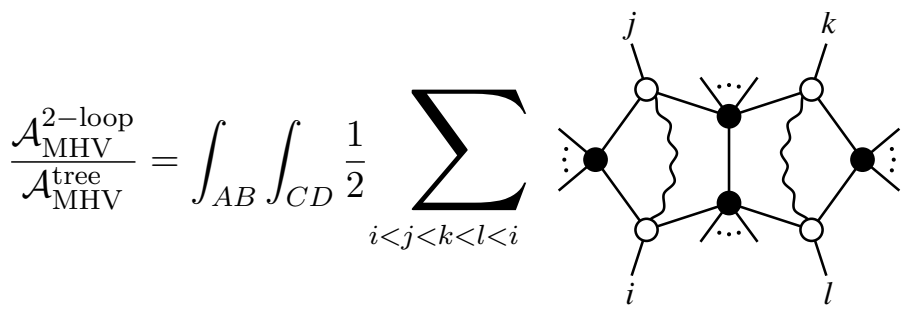

in terms of the chiral double pentagon integrand

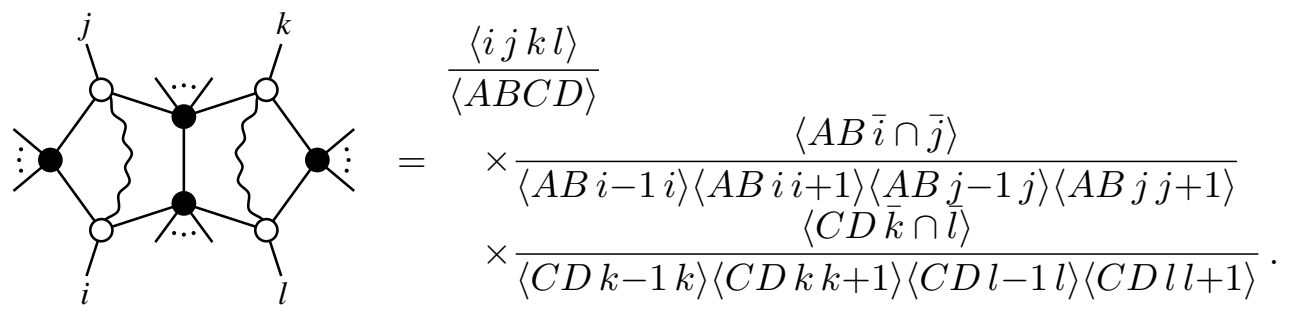

The numerator factors in (4.2) serve the same purpose as in the one-loop pentagon discussed in the previous section. Each of the two nontrivial numerator factors vanishes 
on half of the leading singularities of the scalar double pentagon integrand; their product is non-zero on one quarter of them. The integrand is normalized to have residue 1 on these leading singularities. The numerator factors also suppress the soft/collinear divergences, rendering the integral finite for generic $i, j, k, l$.

Explicit analytic results for the chiral double pentagon integral have been obtained only for the special case $l=k+2=j+3=i+5$ at $n=6$ [17]. However it is expected that for generic $i, j, k, l$ the integral is expressible as a generalized polylogarithm with a symbol alphabet similar to that described in (3.5) and (3.6). Specifically, the letters appearing in the first entry of the symbol are expected on general physical grounds [14] to be

$$
\langle a a+1 b b+1\rangle, \quad a, b \in\{i-1, i, j-1, j, k-1, k, l-1, l\} .
$$

In the second entry we expect to start seeing additional letters of the type

$$
\langle a \bar{b}\rangle, \quad\langle a(a-1, a+1)(c, c+1)(d, d+1)\rangle
$$

for $a, b \in\{i, j, k, l\}$ and $c, d \in\{i-1, i, j-1, j, k-1, k, l-1, l\}$.

We are less certain about the symbol alphabet for the third and fourth entries of the chiral double pentagon integral. For guidance we rely on the symbol of the full two-loop $n$-point MHV amplitude (4.1), which was determined in ref. [18] and which contains, in its third and fourth entries, letters of the form

$$
\langle a a+1 b c\rangle \text { and }\langle a a+1 \bar{b} \cap \bar{c}\rangle \text {. }
$$

We cannot rule out the possibility that individual chiral double pentagon integrals might have an even larger symbol alphabet, with nontrivial cancellation in the sum (4.1). Indeed some cancellation is known to occur: the final entry of the symbol of an MHV amplitude is always of the form $\langle a \bar{b}\rangle[18]$, but we do not expect this to be true for each individual chiral double pentagon integral.

\subsection{Landau singularities}

To find the leading Landau singularities of the double pentagon we must solve for $A B, C D$ which put all nine propagators on-shell. The loop rule (2.3) plays no role in this case for the same reason discussed under (3.7). The eight propagators

$$
\begin{aligned}
\langle A B i-1 i\rangle & =\langle A B i i+1\rangle=\langle A B j-1 j\rangle=\langle A B j j+1\rangle=0 \\
\langle C D k-1 k\rangle & =\langle C D k k+1\rangle=\langle C D l-1 l\rangle=\langle C D l l+1\rangle=0
\end{aligned}
$$

are put on shell just as in (3.8), by

$$
(A, B)=(i, j) \text { or } \bar{i} \cap \bar{j} \quad \text { and } \quad(C, D)=(k, l) \text { or } \bar{k} \cap \bar{l} .
$$

It remains only to set the ninth propagator $\langle A B C D\rangle$ to zero, but in light of (4.7) this becomes a constraint on the external kinematics:

$$
\langle i j k l\rangle\langle i j \bar{k} \cap \bar{l}\rangle\langle\bar{i} \cap \bar{j} k l\rangle\langle\bar{i} \cap \bar{j} \bar{k} \cap \bar{l}\rangle=0 .
$$


The Landau equations for the double pentagon admit a leading solution only on the locus in kinematic space where (4.8) is satisfied.

At sub-leading order there are two possible topologies. If the $\alpha$ associated to the internal propagator $\langle A B C D\rangle$ is set to zero then we simply get two kissing boxes of type (d) in figure 2, whose Landau singularities, according to (2.10), lie on the intersection of

$$
\langle i \bar{j}\rangle\langle\bar{i} j\rangle=0 \quad \text { and } \quad\langle k \bar{l}\rangle\langle\bar{k} l\rangle=0 .
$$

On the other hand if the $\alpha$ associated to one of the eight propagators displayed in (4.6) is set to zero then the diagram degenerates to a pentagon-box. Suppose for example that we collapse the edge $(i, i+1)$. Then the $A B$ loop becomes a 3 -mass box whose leading Landau singularity is at

$$
\langle j(j-1, j+1)(i, i-1) C D\rangle=0
$$

according to (2.8). Meanwhile $C D$ is determined by the same four equations on the second line of $(4.6)$ to be $(C, D)=(k, l)$ or $(C, D)=\bar{k} \cap \bar{l}$. This particular sub-leading Landau singularity therefore lives on the locus

$$
\langle j(j-1, j+1)(i, i-1)(k, l)\rangle\langle j(j-1, j+1)(i, i-1) \bar{k} \cap \bar{l}\rangle=0 .
$$

Altogether there are a total of eight such sub-leading singularities:

(SLLS)

$$
\begin{aligned}
& \langle j(j-1, j+1)(i-1, i)(k, l)\rangle\langle j(j-1, j+1)(i-1, i) \bar{k} \cap \bar{l}\rangle=0, \\
& \langle j(j-1, j+1)(i, i+1)(k, l)\rangle\langle j(j-1, j+1)(i-1, i) \bar{k} \cap \bar{l}\rangle=0, \\
& \langle i(i-1, i+1)(j-1, j)(k, l)\rangle\langle j(j-1, j+1)(i-1, i) \bar{k} \cap \bar{l}\rangle=0, \\
& \langle i(i-1, i+1)(j, j+1)(k, l)\rangle\langle j(j-1, j+1)(i-1, i) \bar{k} \cap \bar{l}\rangle=0 .
\end{aligned}
$$

and four more of the same type but with $i j$ and $k l$ exchanged.

At sub-sub-leading order we can have a triangle-pentagon or a double-box. The former can be obtained by further collapsing the $A B$ integral from a box, as we have just discussed, down to a triangle. Using (2.6) we find a total of eight non-trivial triangle-pentagon singularities:

$\left(\mathbf{S}^{2} \mathbf{L L S}\right)$

$$
\begin{aligned}
& \langle i i+1 j-1 j\rangle\langle j-1 j k l\rangle\langle k l i i+1\rangle\langle j-1 j \bar{k} \cap \bar{l}\rangle\langle\bar{k} \cap \bar{l} i i+1\rangle=0, \\
& \langle i-1 i j-1 j\rangle\langle j-1 j k l\rangle\langle k l i-1 i\rangle\langle j-1 j \bar{k} \cap \bar{l}\rangle\langle\bar{k} \cap \bar{l} i-1 i\rangle=0, \\
& \langle i i+1 j j+1\rangle\langle j j+1 k l\rangle\langle k l i i+1\rangle\langle j-1 j \bar{k} \cap \bar{l}\rangle\langle\bar{k} \cap \bar{l} i-1 i\rangle=0, \\
& \langle i-1 i j j+1\rangle\langle j j+1 k l\rangle\langle k l i-1 i\rangle\langle j-1 j \bar{k} \cap \bar{l}\rangle\langle\bar{k} \cap \bar{l} i-1 i\rangle=0,
\end{aligned}
$$

and again four more of the same type but with $i j$ and $k l$ exchanged. We have included on this list only those degenerations which lead to a three-mass triangle diagram. As discussed earlier, two-mass triangles admit solutions to the Landau equations for generic kinematics.

Next we consider the sub-sub-leading singularities of double box type. Suppose we collapse the edges $(i, i+1)$ and $(l-1, l)$. The three-mass box on the right has a leading 
Landau singularity on the locus $\langle k(k-1, k+1)(l, l+1) A B\rangle=0$. Taking this condition together with the three remaining propagators

$$
\langle A B i-1 i\rangle=\langle A B j-1 j\rangle=\langle A B j j+1\rangle=\langle k(k-1, k+1)(l, l+1) A B\rangle=0,
$$

we see that the left box is also of three-mass type, specified by the four lines $(i-1, i)$, $(j-1, j),(j, j+1)$ and, with the help of $(2.14), k(k-1, k+1)(l, l+1)=\bar{k} \cap(k, l, l+1)$. This three-mass box has its leading Landau singularity on the locus

$$
\langle j(j-1, j+1)(i-i, i) \bar{k} \cap(k, l, l+1)\rangle=\langle\bar{j} \cap(i-1, i, j) \bar{k} \cap(k, l, l+1)\rangle=0 .
$$

The double pentagon has a total of 16 sub-subleading singularities of this type, given by:

$\left(\mathbf{S}^{2} \mathbf{L L S}\right)$

$$
\begin{aligned}
& \langle\bar{i} \cap(i, j-1, j) \bar{l} \cap(k, k+1, l)\rangle=0, \\
& \langle\bar{i} \cap(i, j, j+1) \bar{l} \cap(k, k+1, l)\rangle=0, \\
& \langle\bar{i} \cap(i, j-1, j) \bar{l} \cap(k-1, k, l)\rangle=0, \\
& \langle\bar{i} \cap(i, j, j+1) \bar{l} \cap(k-1, k, l)\rangle=0,
\end{aligned}
$$

and the same with $i \leftrightarrow j$ or $k \leftrightarrow l$.

At $\mathrm{sub}^{3}$-leading order we can have singularities coming from triangle-box topologies. There are a total of 32 degenerations of this type involving a three-mass triangle and a three-mass box, with Landau singularities at the following loci in kinematic space:

$\left(\mathbf{S}^{3} \mathbf{L L S}\right)$

$$
\begin{aligned}
& \langle i i+1 j-1 j\rangle\langle k(k-1, k+1)(l, l+1)(j-1, j)\rangle\langle k(k-1, k+1)(l, l+1)(i, i+1)\rangle=0, \\
& \langle i i+1 j j+1\rangle\langle k(k-1, k+1)(l, l+1)(j, j+1)\rangle\langle k(k-1, k+1)(l, l+1)(i, i+1)\rangle=0, \\
& \langle i-1 i j-1 j\rangle\langle k(k-1, k+1)(l, l+1)(j-1, j)\rangle\langle k(k-1, k+1)(l, l+1)(i-1, i)\rangle=0, \\
& \langle i-1 i j j+1\rangle\langle k(k-1, k+1)(l, l+1)(j, j+1)\rangle\langle k(k-1, k+1)(l, l+1)(i-1, i)\rangle=0,
\end{aligned}
$$

then four more like these but with $(l-1, l)$ instead of $(l, l+1)$, and then another eight given by taking $k \leftrightarrow l$, and finally another sixteen given by those described so far but with $i j$ and $k l$ exchanged.

We could go further down to degenerations involving bubbles, or the triangle-triangle graph, but we will stop here as we have already encountered in the Landau singularities seen so far all of the four-brackets which are known to appear in the symbol of the two-loop MHV amplitudes. In fact we expect that all triangle-triangle and bubble-* diagrams to be either fully singular or to have Landau singularities on the vanishing loci of four-brackets which already appear.

\subsection{Summary and discussion}

The chiral double pentagon integral has quite a long laundry list of Landau singularities. Actually, as we confess in the following section, there are additional classes of singularities that we have not even looked at. It is however clear that all of the symbol entries of 
the two-loop MHV amplitudes, shown in (4.3), (4.4) and (4.5), do vanish on loci where the Landau equations admit solutions. It is an important question for future work to discover whether it is possible to make a stronger statement explaining why the various other Landau singularities do not seem to manifest themselves as symbol entries.

In particular the $S^{2} L L S$ of double box type, shown in (4.16), involve more complicated four-brackets than those which appear in actual two-loop MHV amplitudes. These brackets bear a resemblance to some of the more complicated cluster $\mathcal{A}$-coordinates which appear in the $\operatorname{Gr}(4, n)$ Grassmannian cluster algebra that is apparently relevant for scattering amplitudes in planar SYM theory [19] (see in particular eq. (6.18)). We comment on the intriguing possibility of a deeper connection between Landau singularities and cluster algebras at the end of the next section.

\section{Conclusion and caveats}

Motivated by the observation of ref. [7] that there should be a close connection between symbol entries and solutions of the Landau equations, in this paper we initiated a study of the Landau singularities of Feynman integrals relevant to one- and two-loop MHV amplitudes in planar SYM theory. On general physical grounds it is expected that a quantity may appear in the symbol of some Feynman integral only if the locus where it vanishes corresponds to some Landau singularity; i.e. only if the Landau equations admit a solution on that locus.

At one loop we found a surprising crisp statement: the sub-sub-leading Landau singularities of the pentagon live on the loci where the first entries of the symbols of the MHV amplitudes vanish, while the sub-leading Landau singularities live on the loci where the second entries vanish. The leading Landau singularity appears as an overall pole in the scalar pentagon integral but is canceled by the numerator factor in the chiral pentagon integral (3.2) whose role is to unit normalize the leading singularity of the integrand.

The analysis of the two-loop Landau equations is more complicated. Although we did verify that all of the symbol entries (4.3), (4.4) and (4.5) which are known to appear in two-loop MHV amplitudes vanish on loci where the Landau equations admit solutions, we also found numerous additional solutions which seem not to have a direct connection with the symbol alphabet. We do not have a satisfactory explanation that accounts for the presence of some Landau singularities as symbol letters, while others are excluded.

However, it is very intriguing to note, by comparing (4.3)-(4.5) to (3.9)-(3.11), that all of the symbol entries of the two-loop MHV amplitudes were already present in our classification of the Landau singularities of the one-loop chiral pentagon integral! The two-loop chiral double pentagon certainly has a large list of new Landau singularities, but none of these make any appearance in the symbol of the two-loop amplitude. It is tempting to speculate that this is connected with the fact that for MHV amplitudes, all possible Yangian invariants are exhausted already at one loop [16]; this means that the $L$-loop MHV integrand has no new leading singularities beyond those which are present at one loop. Clearly it would be interesting to extend our analysis to the NMHV amplitudes and beyond. (The two-loop NMHV integrands were written in ref. [13], and all two-loop integrands were constructed in ref. [20].) 
It is clear that we have only taken the first steps in exploring a potentially fruitful connection between symbology and the Landau equations. In the next few paragraphs we discuss several important caveats regarding our analysis. Properly understanding each of these caveats is an interesting, new research direction.

(1) Perhaps the most important caveat is that the Landau equations themselves are completely insensitive to the numerator structure of a Feynman integral; they only identify the locus in kinematic space where a singularity may occur. Yet we know very well that numerator factors in the integrand of SYM theory are not just some afterthought thrown on top of a product of propagators, they are part and parcel of the deep mathematical structure of the integrand. At one loop we were able, with a little bit of handwaving, to explain away the absence of certain Landau singularities in the symbol by appealing to the numerator factor. Clearly it would be important to have a more systematic understanding of the role of numerator factors in SYM theory vis à vis the Landau singularity analysis.

(2) In the analyses of sections 3 and 4, we have ignored an entire class of branch points called "second-type singularities" [2, 21, 22]. These are not solutions to the Landau equations (2.3) and (2.4). Instead, they are pinch singularities of (2.1) at infinite loop momenta, for which (2.3) and (2.4) need to be modified. However, due to dual conformal symmetry, there is no invariant notion of "infinite momentum" in planar SYM theory, so these second-type singularities should be absent except when using an infrared regulator that breaks the dual conformal symmetry. We therefore expect these singularities to be intimately tied to the infrared divergences of SYM theory, and we believe they are completely captured by the BDS subtraction prescription [23] (which can be implemented in a dual conformal preserving way [24]). In any case, recall that the chiral double pentagon integral is actually finite (and hence dual conformal invariant) except for certain boundary cases in $i, j, k, l$.

In non-dual-conformally invariant theories, second-type singularities are ubiquitous and not directly tied to IR divergences. As a simple example, consider the three-mass triangle integral which evaluates explicitly to $[15,25,26]$

$$
I_{3}=\frac{i}{\sqrt{\Delta}} \sum_{i=1}^{3}\left(\operatorname{Li}_{2}\left(x_{i}\right)-\operatorname{Li}_{2}\left(1 / x_{i}\right)\right), \quad x_{i}=\frac{p_{1}^{2}+p_{2}^{2}+p_{3}^{2}-2 p_{i}^{2}+\sqrt{-\Delta}}{p_{1}^{2}+p_{2}^{2}+p_{3}^{2}-2 p_{i}^{2}-\sqrt{-\Delta}}
$$

where

$$
\Delta=-\left(p_{1}^{2}\right)^{2}-\left(p_{2}^{2}\right)^{2}-\left(p_{3}^{2}\right)^{2}+2 p_{1}^{2} p_{2}^{2}+2 p_{2}^{2} p_{3}^{2}+2 p_{1}^{2} p_{3}^{2}
$$

is the triangle Gram determinant. This integral is IR finite yet has an algebraic branch point at a second-type singularity, when $\Delta=0$.

(3) We were fortunate in our analysis of the one- and two-loop pentagons that the Landau singularities we found always live on the vanishing locus of simple geometric quantities expressible in terms of four-brackets of twistors, and that the symbol entries of the corresponding amplitudes were similarly expressible simply in terms of four-brackets. In general, we should not expect the Landau equation analysis to tell us what the symbol entries are, only where they vanish. The three-mass triangle presented above illustrates a 
more complicated case: the LLS of the triangle are located at $p_{i}^{2}=0$, and there is a secondtype singularity at $\Delta=0$, but the arguments of the dilogarithms, and hence the symbol entries, are much more complicated quantities. Closer inspection of these arguments reveals that they do vanish (or become infinite), as required, precisely on the leading Landau singularity locus where (2.6) is satisfied. However one would have been hard-pressed to guess the exact form of these symbol entries just knowing that the Landau singularities are at $p_{i}^{2}=0$ and $\Delta=0$. In many cases at one loop, the actual symbol entries are understood geometrically $[27,28]$, but there is a shortage of appropriate data on higher-loop integrals. We can hope that planar SYM theory is sufficiently special that it is possible to make a more direct connection between Landau singularities and symbol alphabets.

(4) We should emphasize again that our two-loop Landau equation analysis focused on an individual chiral double pentagon integral, which we compared to the symbol alphabet of the full amplitude given by the sum (4.1). Therefore we must acknowledge the possibility of nontrivial cancellation in the sum, such that some symbol entries appearing in individual double pentagon integrals might drop out of the full amplitude.

(5) Finally, although we consider this very implausible, we should acknowledge the possibility that the amplitude, or individual integrals, might have singularities which are only present in beyond-the-symbol terms.

One of the more speculative motivations for this work was the tantalizing possibility that there might exist a physical principle which could dictate the symbol alphabet for a given integral or amplitude; often in actual calculations one must start with an ansatz. We are still very far from understanding whether such a principle exists or may be useful, but it is worthwhile to explore this question since the payoff could be so great. In particular, all evidence available to date is consistent with the hypothesis that all $n$-point MHV and NMHV amplitudes in planar SYM theory have symbol alphabets consisting of the set of cluster coordinates on the $\operatorname{Gr}(4, n)$ Grassmannian cluster algebra [19]. This has been exploited in some of the recent work calculating remainder and ratio functions [29-36]. It would be very interesting to explore the interplay between the physical principle that the singularities of amplitudes are encoded in the Landau equations, and the empirically observed mathematical fact that their singularities are dictated by cluster algebras.

\section{Acknowledgments}

We are grateful to J. Maldacena for discussions. This work was supported by the US Department of Energy under contract DE-SC0010010 Task A (MS) and Early Career Award DE-FG02-11ER41742 (AV), as well as by the Simons Investigator Award of AV.

Open Access. This article is distributed under the terms of the Creative Commons Attribution License (CC-BY 4.0), which permits any use, distribution and reproduction in any medium, provided the original author(s) and source are credited.

\section{References}

[1] L.D. Landau, On analytic properties of vertex parts in quantum field theory, Nucl. Phys. 13 (1959) 181 [INSPIRE]. 
[2] R.J. Eden et al., The analytic S-matrix, Cambridge University Press, Cambridge U.K. (1966).

[3] G.F. Sterman, An introduction to quantum field theory, Cambridge University Press, Cambridge U.K. (1963).

[4] K.T. Chen, Iterated path integrals, Bull. Amer. Math. Soc. 83 (1977) 831.

[5] A.B. Goncharov, A simple construction of Grassmannian polylogarithms, arXiv:0908.2238 [INSPIRE].

[6] A.B. Goncharov, M. Spradlin, C. Vergu and A. Volovich, Classical polylogarithms for amplitudes and Wilson loops, Phys. Rev. Lett. 105 (2010) 151605 [arXiv:1006.5703] [INSPIRE].

[7] J. Maldacena, D. Simmons-Duffin and A. Zhiboedov, Looking for a bulk point, arXiv: 1509.03612 [INSPIRE].

[8] S. Abreu, R. Britto, C. Duhr and E. Gardi, From multiple unitarity cuts to the coproduct of Feynman integrals, JHEP 10 (2014) 125 [arXiv:1401.3546] [INSPIRE].

[9] S. Abreu, R. Britto and H. Grönqvist, Cuts and coproducts of massive triangle diagrams, JHEP 07 (2015) 111 [arXiv: 1504.00206] [INSPIRE].

[10] N. Arkani-Hamed, F. Cachazo, C. Cheung and J. Kaplan, A duality for the S matrix, JHEP 03 (2010) 020 [arXiv: 0907.5418] [inSPIRE].

[11] R.K. Ellis and G. Zanderighi, Scalar one-loop integrals for QCD, JHEP 02 (2008) 002 [arXiv:0712.1851] [INSPIRE].

[12] A. Hodges, Eliminating spurious poles from gauge-theoretic amplitudes, JHEP 05 (2013) 135 [arXiv: 0905.1473] [INSPIRE].

[13] N. Arkani-Hamed, J.L. Bourjaily, F. Cachazo and J. Trnka, Local integrals for planar scattering amplitudes, JHEP 06 (2012) 125 [arXiv:1012.6032] [INSPIRE].

[14] D. Gaiotto, J. Maldacena, A. Sever and P. Vieira, Pulling the straps of polygons, JHEP 12 (2011) 011 [arXiv:1102.0062] [INSPIRE].

[15] Z. Bern, L.J. Dixon and D.A. Kosower, Dimensionally regulated pentagon integrals, Nucl. Phys. B 412 (1994) 751 [hep-ph/9306240] [INSPIRE].

[16] N. Arkani-Hamed et al., Scattering amplitudes and the positive grassmannian, arXiv: 1212.5605 .

[17] J.M. Drummond and J.M. Henn, Simple loop integrals and amplitudes in $N=4$ SYM, JHEP 05 (2011) 105 [arXiv: 1008.2965] [inSPIRE].

[18] S. Caron-Huot, Superconformal symmetry and two-loop amplitudes in planar $N=4$ super Yang-Mills, JHEP 12 (2011) 066 [arXiv:1105.5606] [INSPIRE].

[19] J. Golden, A.B. Goncharov, M. Spradlin, C. Vergu and A. Volovich, Motivic amplitudes and cluster coordinates, JHEP 01 (2014) 091 [arXiv: 1305.1617] [INSPIRE].

[20] J.L. Bourjaily and J. Trnka, Local integrand representations of all two-loop amplitudes in planar SYM, JHEP 08 (2015) 119 [arXiv:1505.05886] [INSPIRE].

[21] D.B. Fairlie et al., Singularities of the second type, J. Math. Phys. 3 (1962) 594.

[22] D.B. Fairlie et al., Physical sheet properties of second type singularities, Phys. Lett. 3 (1962) 55. 
[23] Z. Bern, L.J. Dixon and V.A. Smirnov, Iteration of planar amplitudes in maximally supersymmetric Yang-Mills theory at three loops and beyond, Phys. Rev. D 72 (2005) 085001 [hep-th/0505205] [INSPIRE].

[24] L.F. Alday, J.M. Henn, J. Plefka and T. Schuster, Scattering into the fifth dimension of $N=4$ super Yang-Mills, JHEP 01 (2010) 077 [arXiv:0908.0684] [INSPIRE].

[25] G. 't Hooft and M.J.G. Veltman, Scalar one loop integrals, Nucl. Phys. B 153 (1979) 365 [INSPIRE].

[26] H.J. Lu and C.A. Perez, Massless one loop scalar three point integral and associated Clausen, Glaisher and L functions, SLAC-PUB-5809 (1992) [INSPIRE].

[27] A. Goncharov, Volumes of hyperbolic manifolds and mixed Tate motives, J. AMS 12 (1996) 569 [alg-geom/9601021].

[28] M. Spradlin and A. Volovich, Symbols of one-loop integrals from mixed Tate motives, JHEP 11 (2011) 084 [arXiv: 1105.2024] [INSPIRE].

[29] L.J. Dixon, J.M. Drummond and J.M. Henn, Bootstrapping the three-loop hexagon, JHEP 11 (2011) 023 [arXiv: 1108.4461] [INSPIRE].

[30] L.J. Dixon, J.M. Drummond and J.M. Henn, Analytic result for the two-loop six-point NMHV amplitude in $N=4$ super Yang-Mills theory, JHEP 01 (2012) 024 [arXiv:1111.1704] [INSPIRE].

[31] L.J. Dixon, J.M. Drummond, M. von Hippel and J. Pennington, Hexagon functions and the three-loop remainder function, JHEP 12 (2013) 049 [arXiv:1308.2276] [INSPIRE].

[32] L.J. Dixon, J.M. Drummond, C. Duhr and J. Pennington, The four-loop remainder function and multi-Regge behavior at NNLLA in planar $N=4$ Super-Yang-Mills theory, JHEP 06 (2014) 116 [arXiv:1402.3300] [INSPIRE].

[33] L.J. Dixon, J.M. Drummond, C. Duhr, M. von Hippel and J. Pennington, Bootstrapping six-gluon scattering in planar $N=4$ super-Yang-Mills theory, PoS(LL2014) 077 [arXiv: 1407 .4724] [INSPIRE].

[34] L.J. Dixon and M. von Hippel, Bootstrapping an NMHV amplitude through three loops, JHEP 10 (2014) 065 [arXiv: 1408.1505] [INSPIRE].

[35] J.M. Drummond, G. Papathanasiou and M. Spradlin, A symbol of uniqueness: the cluster bootstrap for the 3-loop MHV heptagon, JHEP 03 (2015) 072 [arXiv: 1412.3763] [INSPIRE].

[36] L.J. Dixon, M. von Hippel and A.J. McLeod, The four-loop six-gluon NMHV ratio function, JHEP 01 (2016) 053 [arXiv: 1509.08127] [INSPIRE]. 